\title{
Artificial Neural Networks approach to the voltage sag classification
}

\author{
F. Ortiz, A. Ortiz, M. Mañana, C. J. Renedo, F. Delgado, L. I. Eguíluz \\ Department of Electrical and Energy Engineering \\ E.T.S.I.I., Cantabria University \\ Avenida de Castros, 39005 Santander (Spain) \\ Phone: +34-942-201374, Fax: +34-942-201385 \\ E-mail: ortizfa@unican.es
}

\section{Introduction}

In the new open-access and competitive power market, electricity consumers are in a unique position to demand a higher quality of service. The utilities or other power providers have to ensure a high quality of their service to remain competitive and retain/attract the customers. Efficient power quality (PQ) monitoring and analysis system can help to achieve this goal.

PQ monitoring can usually be a complex task involving hardware instrumentation and software packages. The instrumentation entails communication software to accomplish the expected functionality. Various types of intelligent electronic devices can be used for collecting the desired PQ data. Examples include dedicated digital fault recorders and protective relays [1-5].

After PQ data of interest are obtained, a comprehensive PQ assessment can be carried out depending on the purpose of the study. This may include power system and equipment modeling verification, $\mathrm{PQ}$ problem mitigation and optimization, and data analysis [4,5]. In most cases, automated PQ assessment is desirable because manual analysis may be difficult to carry out due to lack of time and special expertise. Specialized software tools can make use of intelligent techniques to automate the PQ assessment for improved accuracy and efficiency [5]. This paper is focusing on automated detection and classification of voltage sags that may facilitate the overall PQ assessment. Intelligent techniques as fuzzy logic or neural networks have been utilized for developing PQ tools. Thus, a neural network approach will be undertaken in our case.

For many applications, the accuracy and efficiency resulting from the existing approaches may not be adequate. Improved accuracy is desirable. Aiming at this goal, this paper proposes a neural network system for making a decision based on the features extracted from voltage measurements.

One of the typical problems in electrical distribution systems are voltage sags. These have a great incidence on the electrical equipment connected to the network in the same area. The sags cause more damages than the rest of disturbances because these have a greater frequency of occurrence. Nowadays the research in this issue is still getting new results. Thus, we will propose here another step forwards in order to know the type of sag.

During sags the voltage suffers a sudden reduction between $90 \%$ and $10 \%$. Immediately after a short period of time the voltage recovers its value. Conventionally the duration of voltage sag are between 10 milliseconds and 1 minute. The voltage sag depth is defined as the difference between the minimum effective value (RMS) during the sag and the reference voltage. Reductions which do not overtake the limit of the $10 \%$ will not be considered as sags.

Nowadays the research in this issue is still getting new results. Thus, we will show here another step forwards in order to implement a fuzzy sag classification based on a DSP measurement platform.

\section{Key words}

Power quality, Voltage sags, Neural Networks, Digital Signal Processing.

\section{Causes of voltage sags}

\section{Shortcircuits and Faults to ground.}

The most common cause of voltage sags are shortcircuits and faults to ground. These cases lead to the more severe voltage falls and to most of the failures in equipment.

One example of voltage sag due to fault is shown below. The case of Fig. 1 is associated with different voltage reductions in two phases while the rms value of the other one is not affected by the perturbation. Different effective values in the three phases are a common characteristic in voltage sags caused by faults. Only shortcircuits in the three phases lead to equal values. Another remarkable feature of these cases is the abrupt fall and recovery of the voltage; associated with the initiation and the compensation, respectively. 


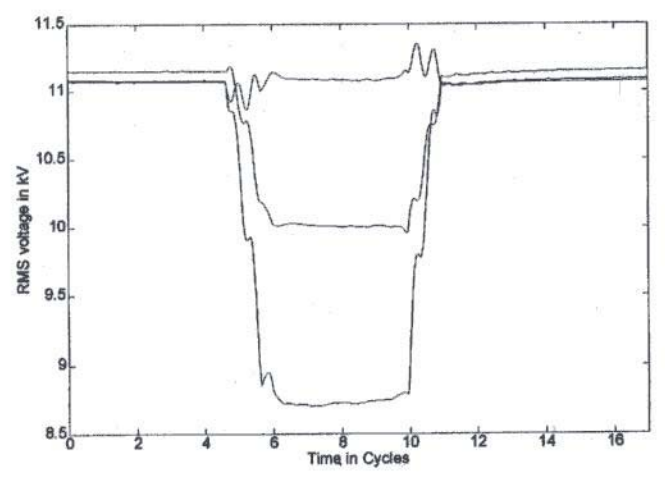

Fig.1 Voltage sag due to a non symmetrical fault.

Motors and Transformers.

Starting current of great induction motors is also a cause of voltage sags in power systems. The duration of this type of disturbance is much longer. Voltage sags due to a motor start show a sudden reduction with a slow recovery depending on ratio torque-speed of the load. Since induction motors are a balanced load, the reduction of the rms voltage is the same for the three phases.

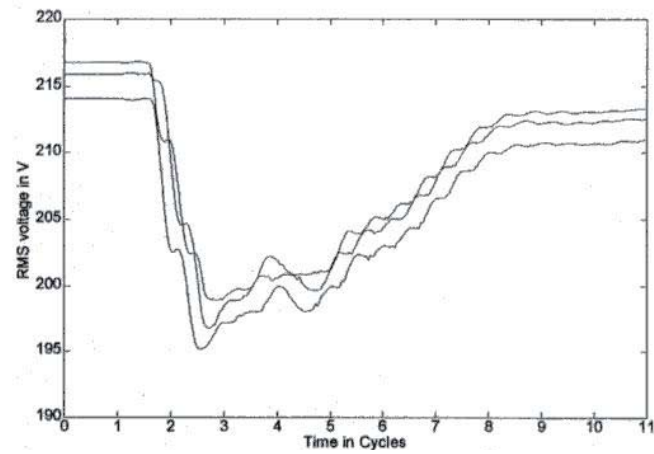

Fig.2 Voltage sag due to a motor starting

Transformers can also provoke voltage sags when they start. The process is characterized by a repetition of short sags with duration of a few milliseconds. These type of events have a great harmonic content. The voltage recovery it is quite slow and it depends on the decomposition of the dc component in the magnetic flux. Different effective values in the three phases are also a common characteristic in voltage sags caused by transformers

\section{Post-Faults.}

A fault is a very severe disturbance of the power system, affecting as much the distribution as the load, and frequently at large distances. However, in this case the voltage recovery is not complete and does not reach the pre-fault value. The two main causes of Post-Faults are induction motors accelerations and transformers saturation.

During faults induction motors reduce speed, increasing gradually the current absorbed from the distribution system and causing a deep voltage reduction. These postfaults currents provoke post-faults voltage sags.

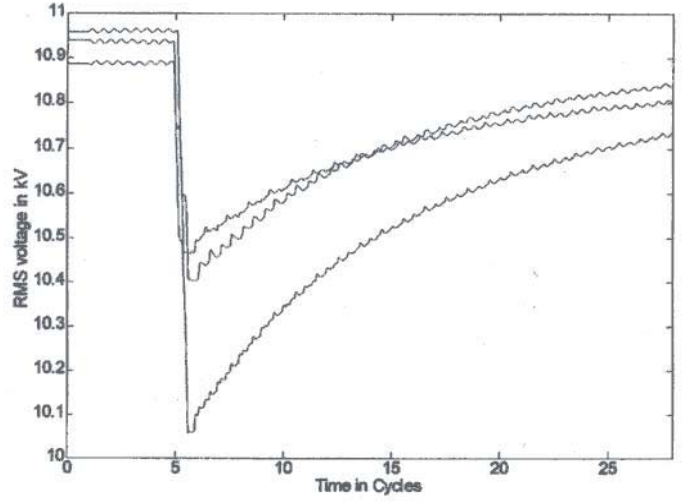

Fig.3 Voltage sag due to a transformer starting

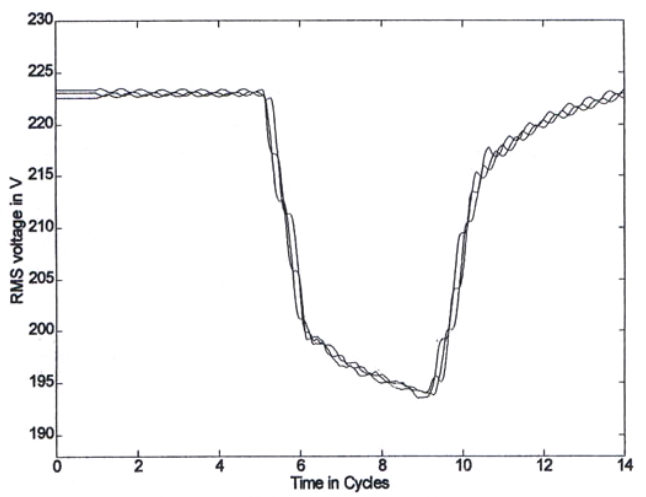

Fig.4 Voltage sag due to a three phase fault

\section{Consequences of voltage sags}

Many devices, whose use is very extended in industrial plants, have become highly sensitive to voltage sags. Between those, variable-speed drives and computers are well known. In addition, these devices are the cause of cosiderable damage due to process outages.

Variable-speed drives are very much implemented in current production systems. They are also considered to cause most process interruptions initiated by voltage sags. These are sensitive to single, two and three-phase sags. Automation systems and computers are a second group reported to cause many process outages due to voltage sags.

Thyristor fed DC motors are other sensitive components. In this case the rectifier is the critical component with regard to voltage-sag susceptibility. Induction motors are also sensitive to voltage sag, mostly with the torque, since it is proportional to the voltage. For example, voltage sags that have a drop higher than $30 \%$ result in a motor torque that is smaller than the load torque, causing a speed decrease that may be unacceptable. Contactors is a widespread method to connect a motor to the supply. Although, their voltage-tolerance curve is similar to those of variable-speed drives, the maximum acceptable voltage drop varies significantly between different brands. 


\section{Classification of voltage sags}

There are several classification methods for voltage sags:

Classification using a magnitude: One of the ways to characterize voltage dips is to use a single voltage-drop magnitude expressed as a percentage of the rated or presag voltage. This magnitude is mostly obtained from the root mean square voltages during the sag. It should be remarked that there is no agreement as to whether the pre-sag or the rated voltage should be used as a reference to calculate the relative voltage drop. In addition, several algorithms exists to obtain these values. Generally, this does not cause a problem since in practice they give equal results for most dips. The characterization using only one magnitude can also be used in three-phase systems where the magnitude of the sag is mostly defined as the largest voltage decrease of all three phases during an event. This characterization is not suitable to predict the outage behavior of sensitive equipment and is mainly used for statistical purposes. In practice, it is often used to compare the number of sags in different countries or regions, generally being described by the System Average RMS Variation Frequency Index (SARFI).

Classification using a magnitude and a time period: Characterizing a sag with one magnitude and one duration uses the root mean square voltages to obtain the sag magnitude. The sag duration is defined as the time in which the voltage is below a certain drop. In the case of a three-phase system, the duration is the longest one in all three phases. This characterization generally overestimate the severity of a dip especially in three-phase systems. In the Figure 5, the dip would be characterized by $t_{d i p}$ and $\mathrm{U}_{\text {dip. }}$

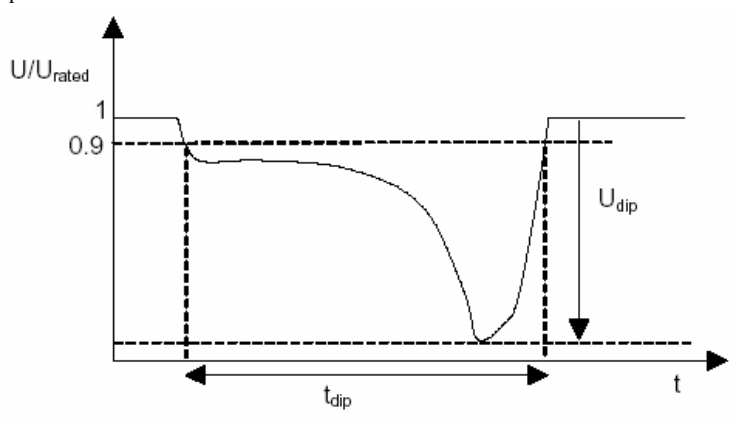

Fig. 5. Characteristic voltage for sags A, B,C and D.

For single-phase equipment, this method can be used to predict outage behavior in a so-called UNIPEDE Table or voltage dip coordination chart with the total di duration on the $\mathrm{x}$-axis and the retained voltage on the $\mathrm{y}$-axis.

Classification using several magnitudes and a time period: in the previous point it has been already mentioned that characterizing sags or dips by only one magnitude and one duration often results in an overestimation of the event, especially for nonrectangular dips, Figure 6. This inaccuracy can be resolved by characterizing a dip using the duration of several magnitude thresholds.

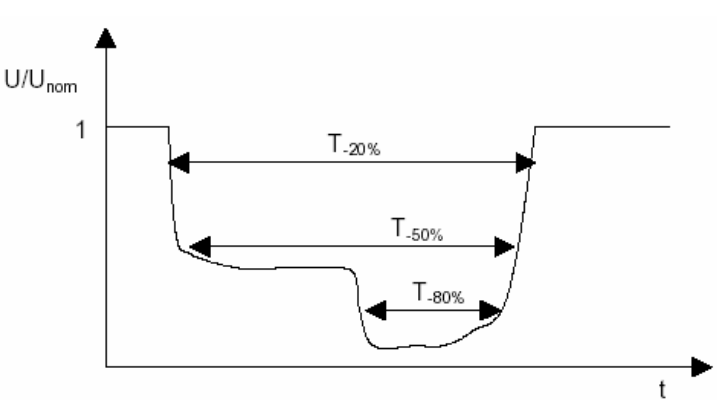

Fig. 6. Characteristic voltage for sags A, B,C and D.

Bollen's classification: In this work the Bollen's classification is used. Seven types of sags are defined which correspond to the possible shortcircuits in the three-phase network and to the sag propagation through transformers. The most common types are A, B, C and D. Their characteristic variation correspond to the following vector diagrams, fig. 7 :
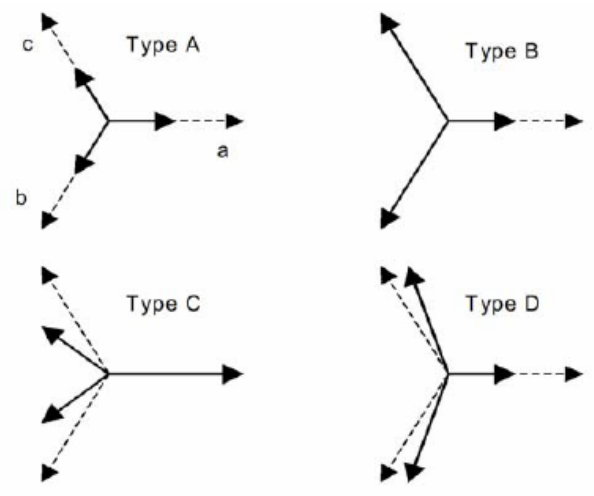

Fig. 7. Characteristic voltage for sags $A, B, C$ and D.

\section{Artificial Neural Networks}

In order to analyze the voltage sags measurement resulting from the PCC, an artificial neural network has been developed using the classification of voltage sags proposed by Bollen. The detection of the type of the sag was simulated with Matlab-Simulink obtaining a satisfactory result. The system has six inputs corresponding to the three voltages, amplitude and angle. The output is the type of sag.

The artificial neuronal networks can be interpreted like an extension of the conventional techniques of statistical recognition of patterns. Frequently, the resolution of a problem of this type is performed naturally, without effort, by humans. However, its solution with computers is more complicated, for that reason, an artificial neuronal network imitates the operation of the brain in order to obtain the same advantages in the processing of information. The use of the neuronal networks offers many properties namely, adaptive learning, parallel operation in real time, tolerance to failures by redundant codification of the information and auto-organization capacity. 
An Artificial Neural Network (ANN) is an informationprocessing tool based on the functional principle of biological neural systems, such as the human brain. The literature identifies four different application areas for neural networks.

\section{Classification}

Classification involves dividing an n-dimensional space into various regions. After this classification, the model can identify to which region a randomly chosen sample belongs. Classification is used in various pattern recognition programs, for example to translate handwritings into ASCII-code.

\section{Prediction}

A neural network can be trained to produce outputs that are expected given a particular input. If a network fits well in modeling a known sequence of values, it can be used to predict future results. The most obvious example for such a predicting network is weather forecasting.

\section{Clustering}

Clustering is used if very complicated data has to be analyzed for which no obvious way exists to classify them into different categories. Neural networks can be used to identify special features of these data and classify them into different categories without prior data knowledge.

\section{Association}

A neural network can be trained to remember a number of patterns. If a distorted version of a particular pattern is presented, the network associates it with the closest one in its memory and returns the original version of that particular pattern. Association is mainly useful for restoring noisy data.

Backpropagation is the type of neuron that is used in the present work. It was created generalizing the multiplelayer networks of Widrow-Hoff. An elementary neuron with $\mathrm{R}$ inputs is shown below. Each input is loaded with the appropriate weight $\mathrm{W}$. The sum of the loaded inputs forms the input to transference function $\mathrm{f}$. The neurons can use any diferenciable transference function $\mathrm{f}$ in order to generate its output.

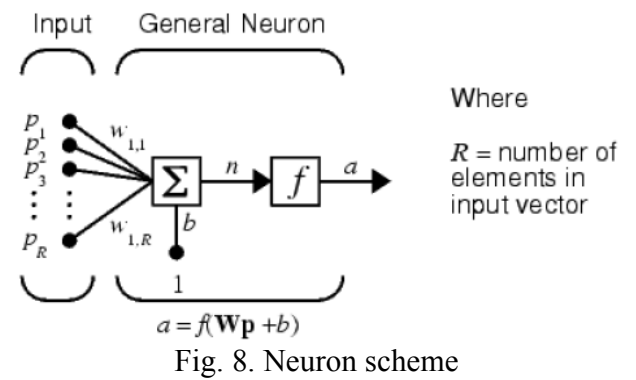

As it was said previously, in this work a Feedforwardbackpropagation neural network has been chosen. Several reasons justify this option:

- Firstly, the authors do not have real data, which makes impossible the learning process by itself.
- On the other hand, we know the equations corresponding to the Bollen's Classification. Thus, it is possible prepare all the cases.

- In addition, the type of output has been determined and this could be related to the input. In this way, the training process is easily controlled.

For the development of the Neuron the Neural Network Toolbox of Matlab is used. Once chosen the type of neuron, it must be trained for the recognition of the different types of voltage sags. There are generally four steps in the training process:

1. Construction of the training data.

2. Design of the network object.

3. Network Training.

4. Simulation of the network respond to new inputs.

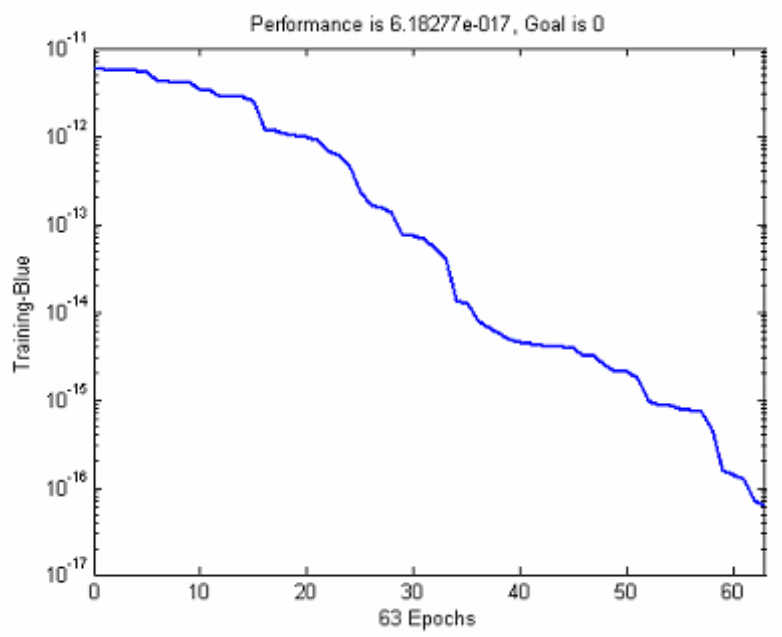

Fig. 9. Training Evolution Error.

\section{Simulation of the voltage sags classification}

The different types of sags are introduced in the neuron and each case is recognized. Here, it was necessary to use the Power Systems Toolbox of Matlab-Simulink.

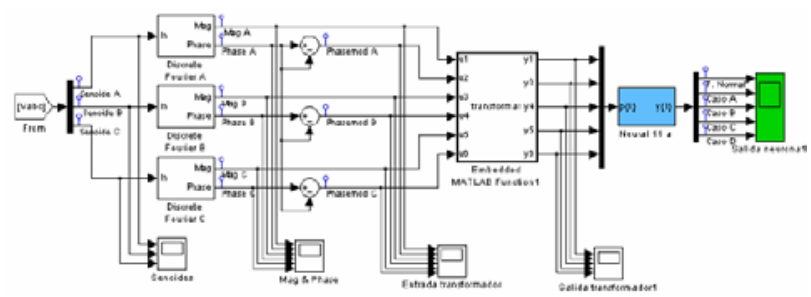

Fig. 10. Simulation of the neural network performance.

In Fig. 12, the output given by the neural network, when different types of sags are introduced, is shown.

\section{Laboratory Setup}

Observing that the algorithm works well, the practical implementation in a real measurement platform was undertaken. Thus, the previous matlab file (.fis) had to be converted to $\mathrm{C}$ code. 
The set-up consists of a device capable of generating the different types of voltage sags, and an resistive-inductive load. A measurement platform DSP based was inserted in this simple system. Their voltage readings were analyzed by means of the neural network, obtaining the type of the sag generated.

The complete laboratory setup can be observed in fig. 11 .

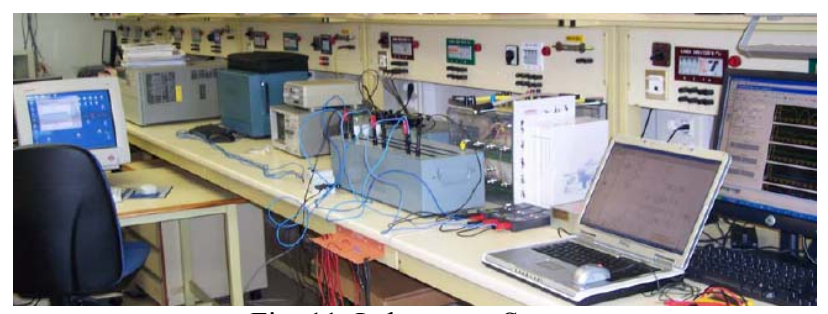

Fig. 11. Laboratory Setup.

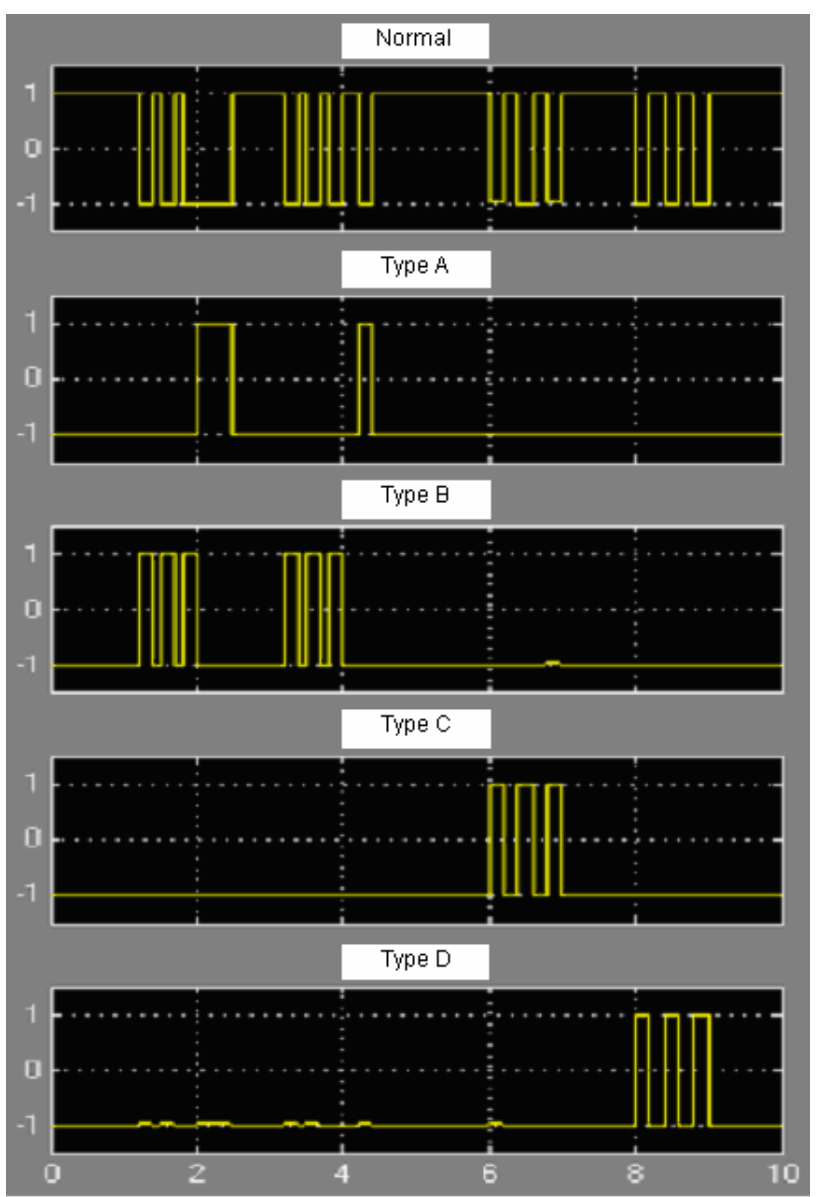

Fig. 12. Output of the neural network simulation.

Once, the neural network proposed is implemented in the DSP measurement platform, the same results obtained in the simulation are found, fig. 13.

\section{Conclusions}

An artificial neural network system has been presented in this paper for detecting and classifying voltages sags. By means of a training process, one could characterize completely a sag. The four more common types proposed by Bollen's classification; A, B, C and D were considered. The inputs into the system are the voltage measurements resulting from the PCC.

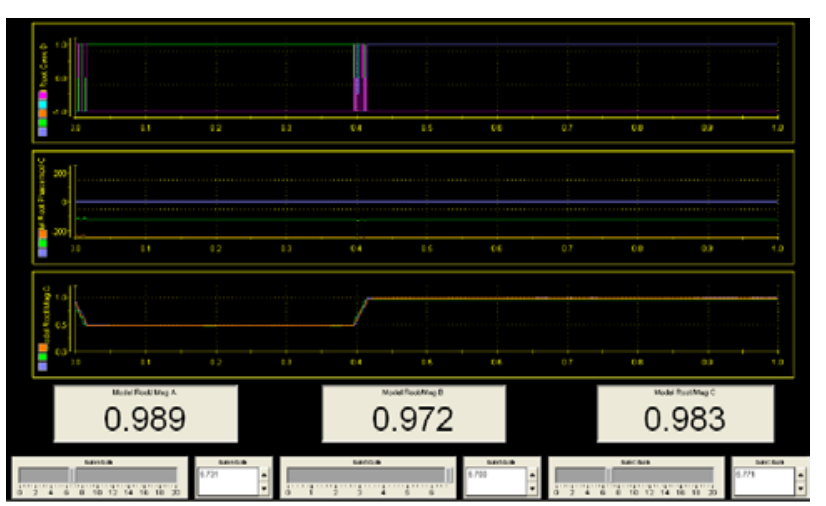

Fig. 13. DSP performance with the neural network implemented.

The algorithm was first tested by simulation with the interface of the Matlab Neural Networks Toolbox. Once the code run properly, it was translated into $\mathrm{C}$ code in order to be implemented in a DSP based measurement platform, obtaining a satisfactory result.

These concepts are easily programmable in current energy meters. In addition, the registration of this information can be very useful for invoicing purposes.

\section{Acknowledgement}

The authors would like to thank the support of the Spanish Government under the CICYT research project DPI2002-04416-C04-01.

\section{References}

[1] IEEE Project 1346 Working Group, Electric power system compatibility with industrial process equipment. Part 1. Voltage sags. IEEE Industrial and Commercial Power Systems Technical Conference, Irvine, CA, USA, May 1-5; 1994. p. 261-6.

[2] IEEE Std 1159-1995, IEEE recommended practice for monitoring electric power quality. New York: IEEE; 1995.

[3] Kezunovic M, Heydt G, Schilleci J, Lambert-Torres G, Key T, Kreis D. Intelligent system applications to power quality and substation automation. Panel Session at the IEEE Power Engineering Society Summer Meeting, Edmonton, Alberta, Canada, July 18-22, vol. 1.; 1999. p. 468-75.

[4] Kezunovic M, Liao Y. Use of intelligent techniques in the power quality assessment applications. International Conference of Intelligent Systems Applications to Power Systems, 1SAP, 2001. Budapest, Hungary, June 18-21 2001.

[5] Liao Y. Automated analysis of power quality data and transmission line fault location. $\mathrm{PhD}$ Dissertation. Texas A\&M University; 2000.

[6] M. Bollen, "Understanding power quality problems : voltage sags and interruptions".

[7] M. Didden et all, "How to connect a voltage sagmeasuring device: Phase-to-Phase or Phase-toNeutral". IEEE transactions on power delivery, vol. 18, no 3, pp. 937-944, July. 